\title{
The association and predictive value analysis of metabolic syndrome on diastolic heart failure in patients at high risk for coronary artery disease
}

\author{
Zi-Hui Tang ${ }^{1}$, Fangfang Zeng ${ }^{2}$, Zhongtao $\mathrm{Li}^{1}$, Yibing $\mathrm{Si}^{2^{*}}$ and Linuo Zhou ${ }^{1 *}$
}

\begin{abstract}
Background: The purpose of the present study was to evaluate the effect and predictive value of metabolic syndrome (MetS) and its components on diastolic heart failure (DHF) in patients at high risk for coronary artery disease (CAD).

Materials and methods: We enrolled 261 patients with normal left ventricular ejection fraction ( $\geq 50 \%)$ who were scheduled to undergo coronary angiography for suspected myocardial ischemia. They were categorized into three groups (non-MetS, pre-MetS and MetS) based on the number of MetS criteria. Echocardiography was used to assess left ventricular (LV) diastolic function. The association between MetS and DHF was assessed by multivariate logistic regression (MLR) analysis (non-DHF patients as reference group) after controlling for confounders. The predictive performance of the MetS severity score (MSS) was evaluated using the area under the receiver-operating characteristic curve (AUC).
\end{abstract}

Results: A tendency toward increased DHF prevalence with increasing MSS was found ( $p<0.001)$. MLR analysis showed that in patients with an MSS of 1, the odds ratio (OR) of DHF was 1.60 (95\% confidence interval-Cl, 1.19-2.16; $p=0.02)$ compared to non-DHF patients; in patients with MSS $\geq 4$, the OR was $6.61(95 \% \mathrm{Cl}, 4.90-8.90$; $\mathrm{p}<0.001)$ compared to non-DHF patients. MSSs strongly predicted DHF (AUC $=0.73,95 \% \mathrm{Cl}, 0.66-0.78, \mathrm{p}<0.001)$. MLR with MetS components as binary variables showed that blood pressure (BP) and triglycerides (TGs) were significantly associated with DHF ( $P=0.001$ and 0.043 , respectively).

Conclusion: Our findings signify that MetS and its components of BP or TG were associated with DHF in high-risk CAD patients. DHF prevalence tends to increase with increasing MSS that has a high value in predicting DHF in high-risk CAD patients.

Keywords: Metabolic syndrome, Diastolic heart failure, High-risk patients, Association, Coronary artery disease

\section{Background}

Metabolic syndrome (MetS) is a global health concern and refers to a constellation of the risk factors of cardiovascular disease, including obesity, abdominal fat distribution, disorders of glucose and lipid metabolism and hypertension (HT) [1]. The burden of MetS is likely to continue to rise, largely due to the decrease in physical

\footnotetext{
*Correspondence: zihui.albertang@gmail.com; dr.zhoulinuo@gmail.com ${ }^{2}$ Department of Cardiology, Huashan Hospital of Fudan University, NO. 12 Wulumuqi Mid Road, Building O\#, Jing'an District, Shanghai 200040, China 'Department of Endocrinology and Metabolism, Huashan Hospital of Fudan University, NO. 12 Wulumuqi Mid Road, Building O\#, Jing'an District, Shanghai 200040, China
}

activity and increase in obesity in our society [2]. Diastolic heart failure (DHF) refers to a decline in the performance of one or both ventricles of the heart during diastole and is characterized by elevated diastolic pressure in the left ventricle (LV), despite an essentially normal end-diastolic volume [2]. Studies have suggested that the morbidity and mortality of DHF are similar to those of systolic heart failure [3,4]. DHF is a powerful and independent predictor of death [5]. DHF can be attributed to multiple factors that are mainly linked to metabolic disturbances $[4,5]$. Some components of MetS- such as hypertension and fasting blood glucose are strongly associated with DHF, which leads to 
stiffening of the LV, resulting in diastolic dysfunction $[4,5]$. Moreover, hypertension, diabetes mellitus (DM) and obesity have been found to adversely affect cardiac structure and function [6,7].

Patients who are at a high risk for coronary artery disease (CAD) characteristically have HT, DM and hyperlipidemia. MetS and DHF tend to be co-prevalent in high-risk CAD patients, who account for more than half of the patients hospitalized in departments of cardiovascular disease [8]. High-risk CAD patients with DHF have been found to have high morbidity and mortality. It is important to clarify the relationship between MetS and DHF in high-risk CAD patients, as this information can be of benefit to clinicians in the prediction, prevention and treatment of DHF. However, the extent to which the clustering of MetS components predicts DHF in highrisk CAD patients is unknown. In addition, the effects of MetS and its components on DHF have not been well characterized in this subgroup of patients. The purpose of the present study was to evaluate the effect and predictive value of MetS and its components on DHF in high-risk CAD patients.

\section{Materials and methods Study population}

Two hundred and sixty-one consecutive symptomatic or asymptomatic Chinese patients who had normal LV ejection fraction (LVEF; $250 \%$ ) and were scheduled to undergo coronary angiography for suspected myocardial ischemia were recruited between February 2009 and March 2011 at the Huashan Hospital of Fudan University, China. Patients were excluded from the study to eliminate potential confounding factors that may have influenced heart function. The exclusion criteria were as follows: (1) history or findings of systolic heart failure $(\mathrm{LVEF} \leq 45 \%)$, significant valvular heart disease (i.e., more than a mild valvular insufficiency or stenosis), hyperthyroidism or hypothyroidism and dilated or hypertrophic cardiomyopathy, (2) pregnancy or lactation and/or (3) a major systemic illness, such as systemic lupus erythematosus. Written consent was obtained from all patients before the study, which was performed in accordance with the ethical standards laid down in Declaration of Helsinki and approved by the Medicine Ethical Committee of Fudan University.

MetS was diagnosed according to the updated $\mathrm{Na}$ tional Cholesterol Education Program/Adult Treatment Panel III criteria (WHO Western Pacific Region obesity criteria) in individuals meeting three or more of the following [9]: (1) central obesity, defined using ethnicityspecific values as a waist circumference (WC) of $\geq 90 \mathrm{~cm}$ in men or $\geq 80 \mathrm{~cm}$ in women; (2) raised triglyceride (TG) levels, $>150 \mathrm{mg} / \mathrm{dl}(1.7 \mathrm{mmol} / \mathrm{l})$ or specific treatment for this lipid abnormality; (3) reduced high-density lipoprotein (HDL) cholesterol, $<40 \mathrm{mg} / \mathrm{dl}(1.03 \mathrm{mmol} / \mathrm{l})$ in men and $<50 \mathrm{mg} / \mathrm{dl}(1.29 \mathrm{mmol} / \mathrm{l})$ in women or specific treatment for this lipid abnormality; (4) raised blood pressure (BP), systolic BP $>130 \mathrm{~mm} \mathrm{Hg}$ or diastolic $\mathrm{BP}>85 \mathrm{~mm} \mathrm{Hg}$ or treatment for previously diagnosed HT; and (5) raised fasting plasma glucose (FPG) level, $>100 \mathrm{mg} / \mathrm{dl}(>5.6 \mathrm{mmol} / \mathrm{l})$ or previously diagnosed type 2 DM. Patients with raised FPG levels were strongly recommended to undergo an oral glucosetolerance test. In addition, if the body mass index (BMI) was $>30 \mathrm{~kg} / \mathrm{m}^{2}$, central obesity was assumed, and WC did not need to be measured. For analysis, the study subjects were grouped according to the number of MetS criteria they satisfied: non-MetS (0 criteria), pre-MetS (1-2 criteria) and MetS ( $\geq 3$ criteria). MetS severity was scored on a scale of 0 to 4 according to the number of MetS components present. Three subjects met all five MetS criteria, and their MetS severity score (MSS) was set to 4 .

The subjects were interviewed for the documentation of medical histories, medications, history of smoking habits, laboratory assessment of cardiovascular disease risk factors and standardized echocardiographic examination. BMI was calculated as the weight in kilograms divided by the square of height in meters. SBP and DBP values were the means of two physician-obtained measurements on the left arm of the seated participant. HT was diagnosed if the BP was $\geq 140 / 90 \mathrm{~mm} \mathrm{Hg}$ and/or the patient was undergoing antihypertensive therapy. DM was diagnosed on the basis of the oral glucose tolerance test and either a glycosylated hemoglobin (HbAlc) level of $\geq 6.5 \%$ or the use of insulin or hypoglycemic medications. CAD was diagnosed if any one of the following were present: (1) history and/or treatment for angina and/or myocardial infarction; (2) history of coronary artery revascularization procedures and/or coronary angiography with $\geq 50 \%$ stenosis in one or more of the major coronary arteries; and (3) regional wall-motion abnormalities on rest echocardiography.

\section{Laboratory assays}

Peripheral venous blood samples were collected in tubes in the fasting state in all subjects. The blood was centrifuged at $3000 \mathrm{rpm}$ for $10 \mathrm{~min}$ for plasma separation and immediately used to measure biomarkers. FPG was measured using the glucose oxidase procedure; HbA1c was measured using ion-exchange high-performance liquid chromatography (HPLC; Bio-Rad, Hercules, CA, USA). Serum total cholesterol (TC), HDL cholesterol, TGs, creatinine (Cr) and uric acid (UA) levels were measured using an enzymatic method with a chemical analyzer (Hitachi 7600-020, Tokyo, Japan). Low-density lipoprotein (LDL) cholesterol levels were calculated using the Friedewald formula, and the creatinine clearance rate (Ccr) was calculated using the Cockcroft-Gault 
formula. The day-to-day and inter-assay coefficients of variation at the central laboratory in our hospital for all analyses were between $1 \%$ and $3 \%$.

\section{Echocardiographic measurement}

Echocardiography examinations were performed with a Vingmed System 5 Doppler echocardiographic unit (GE Vingmed Ultrasound, Horten, Norway). Conventional echocardiography measurements were performed according to American Society of Echocardiography guidelines. LV mass (LVM) was calculated using the Devereux formula. The LVM was corrected for body surface area (BSA) to obtain the LVM index (LVMI). The left atrial diameter (LAD) and aortic root dimension (AOD) were also measured. LV systolic function was assessed using the LVEF. Diastolic function was assessed by determining the E-to-A ratio (E/A) and deceleration time (DT), where E and A represent the early and late ventricular filling velocities, respectively.

We used the definition of DHF recommended in the European Society of Cardiology guidelines in 2008 [10]. The diagnosis of DHF required the following three conditions: (1) presence of signs and/or symptoms of chronic heart failure meanly involved in shortness of breath, rapid heartbeat, wheezing or abnormal heart sounds and swollen legs; (2) presence of normal or only mildly abnormal LV systolic function (LVEF, 45\%-50\%); and (3) evidence of diastolic dysfunction (abnormal LV relaxation or diastolic stiffness). Diastolic function of the LV was evaluated on the basis of the ventricular filling pattern in patients with heart failure. Normal LV diastolic function was defined as an E/A ratio $>1$ and $160 \mathrm{~ms}<\mathrm{DT}<240 \mathrm{~ms}$. LV diastolic dysfunction was defined as (1) E/A ratio $<1$ and DT $\geq 260 \mathrm{~ms}$ or (2) E/A ratio $>2$ and $\mathrm{DT}<150 \mathrm{~ms}$.

\section{Statistical analysis}

The Kolmogorov-Smirnov test was used to determine whether continuous variables followed a normal distribution. Variables that were not normally distributed were log-transformed to approximate normal distribution for analysis. The results are expressed as the mean \pm SD or median, unless otherwise stated. The characteristics of the subjects according to MetS groups were assessed using one-way analysis of variance (ANOVA) for continuous variables and the $x^{2}$ test for categorical variables. Univariate linear regression was performed to determine the variables associated with DHF and to estimate confounding factors possibly disturbing the relationship between MetS and DHF. Multivariate logistic linear regression (MLR) was carried out to determine the independent contributions of variables to DHF (nonDHF patients as reference group). Potential confounding variables, including age, gender, smoking, LAD, LVMI,
UA, Ccr and Cr, were controlled in the regression model. Variables were entered into the backward stepwise regression models if a $p$ value of $<0.10$ was obtained. The models were re-analyzed after substituting the continuous variables related to all MetS components with their dichotomous counterparts in the models. The predictive performance of the MSS was evaluated using the area under the curve (AUC) in a receiver operating characteristics (ROC) curve. Odds ratios (ORs) with 95\% confidence intervals (CIs) were calculated for the relative risk of MetS with DHF. The results were analyzed using the Statistical Package for Social Sciences for Windows, version 16.0 (SPSS, Chicago, IL, USA). The tests were two-sided, and a $\mathrm{p}$ value of $<0.05$ was considered significant.

\section{Results}

The baseline clinical characteristics of the 261 subjects were grouped according to the type of MetS (Table 1). The total sample included 153 men and 108 women (mean age, $59.56 \pm 14.53$ years), and $24.53 \%$ of these patients were found to have MetS. Gender, height and TC levels were similar among the three MetS groups $(p>0.05)$, while the other demographic parameters and biochemical variables were significantly different $(\mathrm{p}<0.05)$. LVEF did not significantly differ among the three groups, but LAD, DT and LVMI did $(\mathrm{p}<0.05)$. The prevalence of CAD, HT and DM were $30.3 \%, 59.4 \%$ and $39.1 \%$ in the patients, respectively. These three conditions were more prevalent in the MetS group than the other two groups $(\mathrm{p}<0.05)$. Smoking habits were similar among the three groups, while the use of oral medications was significant different $(\mathrm{p}<0.05)$.

\section{Prevalence of DHF in high-risk CAD patients}

The prevalence of DHF was $52.50 \%$ in the total sample and $20.80 \%, 52.3 \%$ and $76.6 \%$ in the non-MetS, preMetS and MetS groups, respectively. DHF prevalence was $71.61 \%, 66.37 \%$ and $69.62 \%$ in patients with HT, DM and CAD, respectively (Figure 1). DHF prevalence was significantly lower in patients without HT (24.76\%) than in patients with HT $(\mathrm{p}<0.001)$. Similar DHF prevalence was found in patients with DM and without DM (41.89\%) and those with and without CAD (45.14\%; $\mathrm{p}<0.001$ for both). In addition, DHF prevalence significantly differed with the MSS (MSS 0, 20.83\%; 1, 41.77\%; 2, 64.28\%; 3, 71.11\%; and 4, 89.47\%; $\mathrm{p}<0.01$; Figure 2). As the MSS increased, the prevalence of DHF also increased ( $\mathrm{p}$ for trend $<0.01$; Figure 2).

\section{MetS severity and DHF}

To estimate the association of MetS severity and other factors with DHF, univariate logistic regression models were developed to include gender, age, height, weight, BMI, WC, SBP, DBP, FPG, TG, HDL, other lipid profiles, 
Table 1 Characteristics of the study population

\begin{tabular}{|c|c|c|c|c|c|}
\hline Variable & Total $(n=261)$ & Non-MetS $(n=48)$ & Pre-MetS $(n=149)$ & MetS $(n=64)$ & $p$ value \\
\hline Age & $59.56 \pm 14.53$ & $51.27 \pm 14.45$ & $59.68 \pm 14.4$ & $65.48 \pm 11.85$ & $<0.001$ \\
\hline Gender (male, \%) & $153(58.6 \%)$ & $25(52.1 \%)$ & $89(59.7 \%)$ & $39(60.9 \%)$ & 0.588 \\
\hline Height & $165.83 \pm 8.11$ & $165.1 \pm 8.45$ & $165.95 \pm 7.77$ & $166.08 \pm 8.71$ & 0.789 \\
\hline Weight & $65.11 \pm 11.74$ & $58.55 \pm 8.49$ & $63.63 \pm 10.87$ & $73.47 \pm 11.34$ & $<0.001$ \\
\hline BMI & $23.56 \pm 3.54$ & $21.38 \pm 2.08$ & $22.97 \pm 3.29$ & $26.55 \pm 3.11$ & $<0.001$ \\
\hline WC & $82.92 \pm 7.82$ & $77.97 \pm 6.213$ & $82 \pm 7$ & $87.04 \pm 7.19$ & $<0.001$ \\
\hline SBP & $128 \pm 18$ & $116 \pm 11$ & $128 \pm 18$ & $136 \pm 18$ & $<0.001$ \\
\hline DBP & $76 \pm 11$ & $72 \pm 7$ & $76 \pm 11$ & $81 \pm 13$ & $<0.001$ \\
\hline$H R$ & $71 \pm 11$ & $66 \pm 11$ & $72 \pm 11$ & $73 \pm 12$ & 0.007 \\
\hline \multicolumn{6}{|l|}{ Laboratory assay } \\
\hline $\mathrm{FPG}$ & $6.02 \pm 2.52$ & $4.83 \pm 0.26$ & $6.11 \pm 2.88$ & $6.73 \pm 2.25$ & $<0.001$ \\
\hline PBG & $8.48 \pm 4.08$ & $6.24 \pm 0.83$ & $8.4 \pm 4.25$ & $9.66 \pm 4.32$ & 0.004 \\
\hline HbAlc & $6.74 \pm 2.13$ & $5.23 \pm 0.42$ & $6.91 \pm 2.36$ & $7.16 \pm 1.97$ & 0.003 \\
\hline $\mathrm{TC}$ & $4.5 \pm 1.12$ & $4.21 \pm 0.81$ & $4.59 \pm 1.13$ & $4.49 \pm 1.26$ & 0.142 \\
\hline TG & $1.61 \pm 1.2$ & $0.98 \pm 0.33$ & $1.49 \pm 0.95$ & $2.34 \pm 1.68$ & $<0.001$ \\
\hline $\mathrm{HDL}$ & $1.14 \pm 0.3$ & $1.3 \pm 0.28$ & $1.16 \pm 0.3$ & $0.99 \pm 0.23$ & $<0.001$ \\
\hline LDL & $2.56 \pm 0.89$ & $2.31 \pm 0.67$ & $2.67 \pm 0.92$ & $2.49 \pm 0.91$ & 0.048 \\
\hline $\mathrm{Cr}$ & $76.11 \pm 25.93$ & $68.04 \pm 14.06$ & $74.84 \pm 22.45$ & $84.78 \pm 35.96$ & 0.002 \\
\hline $\mathrm{Ccr}$ & $85.58 \pm 30.31$ & $91.04 \pm 24$ & $85.46 \pm 31.27$ & $81.95 \pm 31.97$ & 0.300 \\
\hline UA & $0.34 \pm 0.1$ & $0.3 \pm 0.08$ & $0.34 \pm 0.1$ & $0.38 \pm 0.12$ & 0.001 \\
\hline \multicolumn{6}{|c|}{ Echocardiographic measurement } \\
\hline LVEF (\%) & $65 \pm 6$ & $65 \pm 4$ & $66 \pm 4$ & $65 \pm 6$ & 0.426 \\
\hline DT & $217.29 \pm 56.37$ & $196.67 \pm 43.63$ & $214.3 \pm 59.54$ & $239.7 \pm 50.12$ & $<0.001$ \\
\hline LAD & $36.17 \pm 4.81$ & $33.67 \pm 4.07$ & $35.7 \pm 4.45$ & $39.14 \pm 4.75$ & $<0.001$ \\
\hline LVMI & $112.77 \pm 35.91$ & $98.51 \pm 28.23$ & $108.46 \pm 35.72$ & $133.71 \pm 33$ & $<0.001$ \\
\hline DHF (yes, \%) & $137(52.5 \%)$ & $10(20.80 \%)$ & $78(52.3 \%)$ & $49(76.6 \%)$ & $<0.001$ \\
\hline \multicolumn{6}{|c|}{ Past medical history (yes, \%) } \\
\hline$C A D$ & $79(30.3 \%)$ & $6(12.5 \%)$ & $41(27.5 \%)$ & $32(50 \%)$ & $<0.001$ \\
\hline $\mathrm{HT}$ & $155(59.4 \%)$ & $0(0.0 \%)$ & $95(63.8 \%)$ & $60(93.8 \%)$ & $<0.001$ \\
\hline DM & 102(39.1\%) & $0(0.0 \%)$ & $58(38.9 \%)$ & $44(68.8 \%)$ & $<0.001$ \\
\hline Smoking & $75(28.7 \%)$ & $14(29.2 \%)$ & 49(32.9\%) & $12(18.8 \%)$ & 0.106 \\
\hline \multicolumn{6}{|c|}{ Medical therapy (yes, \%) } \\
\hline Anti-hypertension & $140(53.6 \%)$ & $0(0.0 \%)$ & $85(57 \%)$ & $55(85.9 \%)$ & $<0.001$ \\
\hline Hypoglycaemic & $78(29.9 \%)$ & $0(0.0 \%)$ & $45(30.2 \%)$ & $33(51.6 \%)$ & $<0.001$ \\
\hline Anti-lipids & $69(26.4 \%)$ & $5(10.41 \%)$ & $41(27.5 \%)$ & $23(35.9 \%)$ & 0.009 \\
\hline
\end{tabular}

Note: $B M I$ Body mass index, SBP systolic blood pressure, DBP diastolic blood pressure, MetS metabolic syndrome, $H T$ Hypertension, DM Diabetes, FPG fasting plasma glucose, $P B G$ plasma blood glucose, $H b A 1 c$ glycated hemoglobin, $T C$ serum total cholesterol, $H D L$ high-density lipoprotein cholesterol, $T G$ triglyceride, UA uric acid, LDL low density lipoprotein cholesterol, $C c r$ creatinine clearance rate, $C r$ creatinine, $L V M I$ left ventricular mass index, LAD left atrial diameter, DT deceleration time, $L V E F$ left ventricular ejection fraction.

Cr, Ccr, UA, echocardiographic parameters of LV, past medical history and medical therapy (Table 2). The univariate analyses indicated that age, height, $\mathrm{Cr}, \mathrm{Ccr}$, UA, LVMI, CAD, DM, HT, MetS and all its components, except HDL, were significantly associated with DHF ( $\mathrm{p}<0.05$ for all). MLR analysis was carried out to determine the extent to which DHF was associated with MetS severity. MetS remained significantly associated with DHF after adjustments for age, height, Cr, Ccr, UA, LVMI, HT, DM and CAD ( $p=0.023$, data not shown). In patients with an MSS of 1 , the OR of DHF was 1.60 (95\% CI, 1.19-2.16; $\mathrm{p}=0.02$; Table 3) compared to non-DHF patients, while in patients with an MSS $\geq 4$, the OR was $6.61(95 \%$ CI, 4.90-8.90; $\mathrm{p}<0.001)$ 


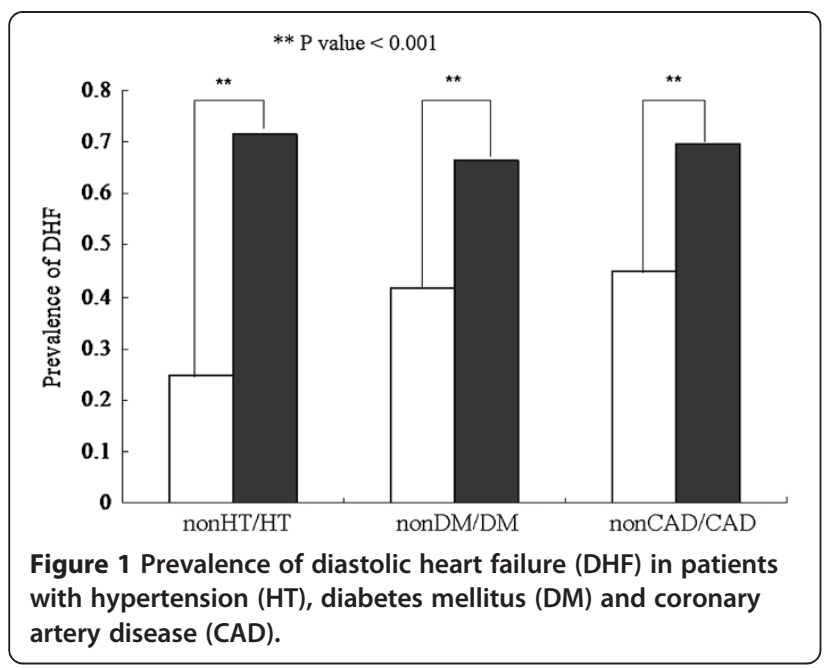

compared to non-DHF patients. To evaluate the predictive performance of MSSs for DHF, the AUC in an ROC curve was calculated. The AUC was $0.726(95 \% \mathrm{CI}$, $0.665-0.787 ; \mathrm{p}<0.001)$, indicating that the MSS strongly predicted DHF (Figure 3).

\section{MetS components and DHF}

MLR analysis with the whole covariate model and backward stepwise regression model with adjustments for confounders were developed to include MetS components as continuous variables. The enter regression model after adjustments for age, Cr, Ccr, UA, LVMI and CAD indicated that DBP was significantly associated with DHF ( $\mathrm{p}=0.022$; data not shown). The backward stepwise regression model showed that age, UA, LVMI and DBP were independently associated with DHF $(\mathrm{p}=0.001,0.041,0.044$ and 0.001 , respectively; data not shown). To reduce the effect of medical therapy, MLR models with adjustments for confounders were developed to include MetS components as binary variables.

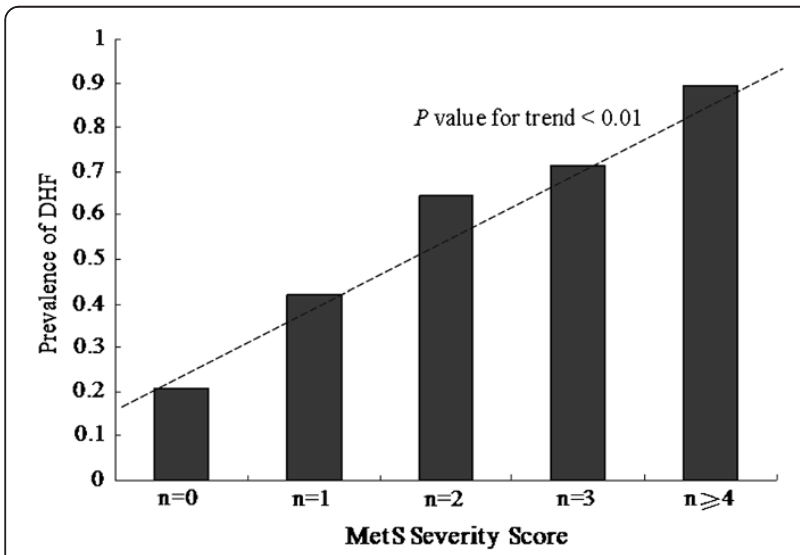

Figure 2 Prevalence of diastolic heart failure (DHF) according to metabolic syndrome (MetS) severity score.
Table 2 Univariate regression analysis for diastolic heart failure

\begin{tabular}{llllll}
\hline Variables & $\boldsymbol{\beta}$ & S.E. & $\boldsymbol{P}$ value & OR & $\mathbf{9 5 . 0 \%}$ C.I. \\
\hline BMI & 0.60 & 0.27 & 0.03 & 1.82 & $1.06-3.14$ \\
SBP & 0.05 & 0.01 & $<0.001$ & 1.06 & $1.04-1.08$ \\
DBP & 0.03 & 0.01 & 0.003 & 1.03 & $1.01-1.06$ \\
FPG & 0.83 & 0.50 & 0.010 & 2.29 & $1.85-6.17$ \\
TG & 0.69 & 0.28 & 0.014 & 2.00 & $1.15-3.47$ \\
HDL & -0.47 & 0.43 & 0.269 & 0.62 & $0.27-1.45$ \\
MetS & 0.78 & 0.13 & $<0.001$ & 2.20 & $1.70-2.84$ \\
Age & 0.86 & 0.11 & $<0.001$ & 2.36 & $1.87-2.99$ \\
CCr & -0.02 & 0.01 & $<0.001$ & 0.97 & $0.97-0.98$ \\
Cr & 0.02 & 0.01 & $<0.001$ & 1.02 & $1.01-1.04$ \\
UA & 1.38 & 0.37 & $<0.001$ & 4.00 & $1.94-8.26$ \\
LVMI & 0.02 & 0.01 & $<0.001$ & 1.02 & $1.02-1.03$ \\
HT & 2.03 & 0.28 & $<0.001$ & 7.66 & $4.36-13.48$ \\
DM & 1.01 & 0.26 & $<0.001$ & 2.73 & $1.65-4.55$ \\
CAD & 1.02 & 0.28 & $<0.001$ & 2.78 & $1.58-4.90$ \\
\hline
\end{tabular}

Note: $M I$ Body mass index, SBP systolic blood pressure, DBP diastolic blood pressure, MetS metabolic syndrome, HT Hypertension, DM Diabetes, FPG fasting plasma glucose, $P B G$ plasma blood glucose, $\mathrm{HbA1c}$ glycated hemoglobin, $T C$ serum total cholesterol, $H D L$ high-density lipoprotein cholesterol, TG triglyceride, UA uric acid, LDL low density lipoprotein cholesterol, $\mathrm{Ccr}$ creatinine clearance rate, $\mathrm{Cr}$ creatinine, LVMI left ventricular mass index, $L A D$ left atrial diameter, $D T$ deceleration time, $L V E F$ left ventricular ejection fraction.

The results showed that BP and TG were significantly associated with DHF ( $\mathrm{p}=0.001$ and 0.043 , respectively in the enter regression model; $\mathrm{p}=0.001$ and 0.038 , respectively in the backward stepwise regression model; Table 4).

\section{Discussion}

We first conducted a case-control study to evaluate the effect of metabolic factors on DHF in Chinese high-risk CAD patients. CAD, HT and DM were more prevalent in patients with MetS. Most of the demographic factors, biochemical characteristics and echocardiographic measurements significantly differed among the three MetS groups. Doppler echocardiography has become a well accepted, reliable, noninvasive tool to measure the

Table 3 Multiple logistic regression analysis for diastolic heart failure, including metabolic syndrome (MetS)

\begin{tabular}{llllll}
\hline MetS severity score & Number & $\boldsymbol{\beta}$ & $\boldsymbol{P}$ value & $\boldsymbol{O} \boldsymbol{R}$ & $\mathbf{9 5 \%} \mathbf{C l}$ \\
\hline 0 (reference) & 48 & 0.0 & - & 1.00 & - \\
1 & 79 & 0.47 & 0.020 & 1.60 & $1.19-2.16$ \\
2 & 70 & 0.94 & $<0.001$ & 2.57 & $1.91-3.46$ \\
3 & 45 & 1.42 & $<0.001$ & 4.12 & $3.06-5.55$ \\
$\geq 4$ & 19 & 1.88 & $<0.001$ & 6.61 & $4.90-8.90$
\end{tabular}

Note: Adjusted for age, gender, serum creatinine, creatinine clearance rate, serum uric acid, left ventricular mass index and coronary artery disease. 




Figure 3 Receiver operative characteristic (ROC) curve showing the performance of metabolic syndrome (MetS) severity score in predicting the prevalence of diastolic heart failure (DHF). Area under the curve $(A \cup C)=0.726$, (95\% confidence interval, $0.665-0.787), p<0.001$

LV diastolic function. In the present study, Doppler echocardiography was used to measure LV diastolic function in order to diagnose DHF.

The main finding of the present study was that MetS strongly and independently predicted DHF in high-risk CAD patients. The prevalence of DHF increased with the severity of MetS. HT, insulin resistance and obesity have been associated with LV diastolic dysfunction or DHF in different populations $[11,12]$. In addition, MetS has been independently correlated with DHF in different

Table 4 Multiple logistic regression analysis for diastolic heart failure (DHF) and components of metabolic syndrome (MetS) as binary variables

\begin{tabular}{lllllll}
\hline Model & Variable & $\boldsymbol{\beta}$ & S.E. & $\boldsymbol{P}$ value & OR & $\mathbf{9 5 \% ~ C l}$ \\
\hline Model 1 & BMI & 0.12 & 0.407 & 0.773 & 1.125 & $0.51-2.5$ \\
& BP & 1.82 & 0.428 & $<0.001$ & 6.197 & $2.68-14.35$ \\
& FBG & 0.47 & 0.364 & 0.201 & 1.592 & $0.78-3.25$ \\
& TG & 0.80 & 0.416 & 0.043 & 2.236 & $1.01-5.05$ \\
& HDL & -0.36 & 0.376 & 0.339 & 0.698 & $0.33-1.46$ \\
Model 2 & Constant & -3.41 & 0.514 & $<0.001$ & & \\
& Age & 0.69 & 0.146 & $<0.001$ & 2.004 & $1.51-2.67$ \\
& UA & 0.93 & 0.488 & 0.045 & 2.546 & $1.08-6.62$ \\
& BP & 1.93 & 0.413 & $<0.001$ & 6.893 & $3.07-15.49$ \\
& TG & 0.81 & 0.388 & 0.038 & 2.236 & $1.05-4.78$ \\
\hline
\end{tabular}

Note: Model 1 adjusted for age, serum creatinine, creatinine clearance rate, serum uric acid, left ventricular mass index and coronary artery disease; Model 2 was the best model. The following variables were entered in step 1: age, serum creatinine, creatinine clearance rate, serum uric acid, left ventricular mass index and coronary artery disease, body mass index, blood pressure, fasting blood glucose, triglycerides and high-density lipoprotein $S E$ standard error, $\mathrm{OR}$ odds ratio, $\mathrm{Cl}$ confidence interval. subgroups such diabetic, non-diabetic or hypertensive patients [13-15]. In the present study, the association between MetS and DHF was observed in both the univariate and multivariate models after adjustment for potential confounders in high-risk CAD patients. Specifically, we found a good association between the MSSs and DHF. To our knowledge, this is the first study to have reported such an association in a population of high-risk CAD patients. In the multivariate analysis, MetS was independently associated with DHF, even after adjustment for potential confounders such as parameters of renal function, LVMI and CAD. This finding is of special importance if the direct relationship between MetS and DHF is considered. The clustering of cardiovascular risk factors in MetS indicates that the multiple complex metabolic reactions involved in glycotoxicity, lipotoxicity, altered insulin signaling, increased cytokine activity and interstitial deposition of triacylglycerol may directly or indirectly impact myocardial function [4,16-20]. Additionally, these metabolic risk factors lead to reduced energy availability, and have an additive, adverse effect on endothelial function [21].

In the present study, the AUC was calculated to show that the MSS strongly predicts DHF. In patients with MSSs of up to 4, the prevalence of DHF was nearly $90 \%$. This finding indicates that the severity of MetS is linked to the progression of DHF. This is one of interesting findings of the present study, which not only supports further studies on the mechanism of DHF but also provides evidence for clinicians to predict DHF in hospitalized patients. However, in the present study, we scored the MetS severity by simply using the number of MetS criteria. We did not consider the weights of the MetS components. For instance, the BP component of MetS makes a greater contribution to DHF. A large-scale, casecontrol study or cohort study with a better method of scoring MetS severity will be conducted to develop a highly sensitive and specific model that uses MetS information to predict DHF. Such a model would facilitate the prevention and treatment of DHF in clinical practice.

Another interesting finding of the present study was that BP and TG were the only MetS components that contributed to DHF. This finding is inconsistent with those of some earlier studies, which had revealed that BMI, SBP, DBP and lipid profiles were significantly associated with diastolic parameters and the structure and functions of the LV $[4,16-18,20]$. In the present study, BMI, FPG and HDL were not significantly associated with DHF. This difference is partly because the contributions of individual MetS components could not be detected in the present study, which had a moderate sample size. Another possible cause is that the present study population differed from those in previous studies; we performed association analysis for MetS and DHF 
in high-risk CAD patients with HT, DM, CAD and hyperlipidemia, which were potential confounders of DHF. In addition, MetS components as continuous variables may not reflect the true values in patients undergoing medical therapy. For example, the FPG values measured in the present study were less than the true values in DM patients using hypoglycemic drugs. However, we focused on metabolic factors associated with DHF in a specific subgroup, high-risk CAD patients. Our findings will provide evidence for clinicians to better understand and treat patients in this specific subgroup. Nevertheless, further studies should explore the effects of MetS on DHF in an exclusive subgroup such as patients with CAD or hyperlipidemia. DBP as a continuous variable was found to be associated with DHF in both the univariate and multivariate models. The results were confirmed in the MLR analysis with $\mathrm{BP}$ as a binary variable. DBP has been found to be an important predictor of LV diastolic dysfunction or DHF [20,22]. DBP can directly influence diastolic function and remodel the LV structure, leading to DHF [23]. In the present study, TG as a binary variable was associated with DHF in both the univariate and multivariate analyses. Other studies have reported similar results $[11,24]$. No consistent results have been found in MLR analyses with TG as a continuous variable. This is partly because high-risk CAD patients were regularly treated with anti-lipids drugs such as statins to prevent events of cardiovascular disease; this may have influenced the true value of TG, making it difficult to determine the effect of TG on DHF. The exact mechanism underlying the association between TG and DHF has not been fully elucidated. In the present study, we did not determine the mechanism via which TG modifies metabolic factors and induces DHF.

Several limitations of the study deserve comment. First, the design of the present study was hospitalbased, which is susceptible to selection bias. Second, the sample size was moderate, limiting its ability to detect significant results. Third, the multiple regression models indicated only a moderate influence of MetS on DHF. Other environmental and genetic factors may contribute to the unexplained variation in DHF prevalence. Fourth, the association between insulin resistance and DHF was not analyzed in the present study. This is because data on fasting blood insulin levels were missing. Furthermore, most of participants were enrolled with the first diagnosis of diastolic heart failure or not. So we did not collect information of the history of diastolic heart failure. Finally, it is important to mention that our study was performed on Chinese individuals, and our findings may not be relevant to people of other ethnicities.

\section{Conclusion}

In conclusion, individuals with MetS frequently have a higher prevalence of DHF. Our findings signify that MetS is an independent predictor of DHF, and BP and TG, as components of MetS, are independently associated with DHF. There is a tendency toward increased prevalence of DHF with increasing MSSs. This supports the hypothesis that MetS is involved in the regulation of DHF progression. The present observations provide evidence that improved metabolic control may coordinately and perhaps even synergistically inhibit the progression of DHF and also provide novel insights into biological functions, in the future.

\section{Abbreviations}

ACEl: Angiotensin-converting enzyme inhibitor; Alb/Cr: Albumin/urinary creatinine ratio; AOD: Aortic root dimension; BMI: Body mass index; BSA: Body surface area; Ccr: Creatinine clearance rate; Cl: Confidence intervals; Cr: Creatinine; DBP: Diastolic blood pressure; DHF: Diastolic heart failure; DM: Diabetes; DT: Deceleration time; E/A: E-to-A ratio; FPG: Fasting plasma glucose; GLM: General linear model; HbAlc: Glycosylated hemoglobin; HDL: High-density lipoprotein cholesterol; HOMA-IR: Homeostasis model assessment insulin resistance estimate; HT: Hypertension; IDF: International diabetes federation; LAD: Left atrial diameter; LDL: Low-density lipoprotein cholesterol; LV: Left ventricle; LVEF: Left ventricular ejection fraction; LVM: Left ventricular mass; LVMI: Left ventricular mass index; MetS: Metabolic syndrome; MLR: Multivariable logistic linear regression; OGTT: Oral glucose tolerance test; OR: Odds ratios; PBG: Postprandial blood glucose; HT: Hypertension; SBP: Systolic blood pressure; TC: Serum total cholesterol; TG: Triglyceride; WC: Waist circumference; UA: Uric acid.

\section{Competing interest}

The authors declare that they have no conflicts of interest.

\section{Authors' contribution}

LZ and YS conceived of the study, and participated in its design and coordination and helped to draft the manuscript. Z-HT participated in the design of the study and performed the statistical analysis, carried out the molecular genetic studies, participated in the sequence alignment

and drafted the manuscript. FZ and ZL carried out the molecular genetic studies, participated in the sequence alignment and drafted the manuscript All authors read and approved the final manuscript.

\section{Acknowledgments}

We thank the grant from China National Grant on Science and Technology to support the study.

\section{Funding}

This study was supported by the grant from China National Grant on Science and Technology (grant number: 30570740).

Received: 29 December 2012 Accepted: 16 June 2013

Published: 24 June 2013

\section{References}

1. Lakka HM, Laaksonen DE, Lakka TA, et al: The metabolic syndrome and total and cardiovascular disease mortality in middle-aged men. JAMA 2002, 288:2709-2716.

2. Aurigemma GP, Gaasch WH: Clinical practice. Diastolic heart failure. N Engl J Med 2004, 351:1097-1105.

3. Bhatia RS, Tu JV, Lee DS, et al: Outcome of heart failure with preserved ejection fraction in a population-based study. N Engl J Med 2006, 355:260-269.

4. Owan TE, Hodge DO, Herges RM, et al: Trends in prevalence and outcome of heart failure with preserved ejection fraction. N Engl J Med 2006, 355:251-259.

5. Mogelvang R, Sogaard P, Pedersen SA, et al: Cardiac dysfunction assessed by echocardiographic tissue Doppler imaging is an independent predictor of mortality in the general population. Circulation 2009, 119:2679-2685. 
6. Chinali $M$, Devereux RB, Howard $B V$, et al: Comparison of cardiac structure and function in American Indians with and without the metabolic syndrome (the Strong Heart Study). Am J Cardiol 2004, 93:40-44.

7. Fang ZY, Najos-Valencia $\mathrm{O}$, Leano $\mathrm{R}$, et al: Patients with early diabetic heart disease demonstrate a normal myocardial response to dobutamine. J Am Coll Cardiol 2003, 42:446-453.

8. de Simone G, Palmieri V, Bella JN, et al: Association of left ventricular hypertrophy with metabolic risk factors: the HyperGEN study. J Hypertens 2002, 20:323-331.

9. Grundy SM, Hansen B, Smith SC Jr, et al: Clinical management of metabolic syndrome: report of the American Heart Association/National Heart, Lung, and Blood Institute/American Diabetes Association conference on scientific issues related to management. Circulation 2004, 109:551-556.

10. Dickstein K, Cohen-Solal A, Filippatos G, et al: ESC guidelines for the diagnosis and treatment of acute and chronic heart failure 2008: the Task Force for the diagnosis and treatment of acute and chronic heart failure 2008 of the European Society of Cardiology. Developed in collaboration with the Heart Failure Association of the ESC (HFA) and endorsed by the European Society of Intensive Care Medicine (ESICM). Eur J Heart Fail 2008, 10:933-989.

11. de las Fuentes L, Brown AL, Mathews SJ, et al: Metabolic syndrome is associated with abnormal left ventricular diastolic function independent of left ventricular mass. Eur Heart J 2007, 28:553-559.

12. Wisniacki $N$, Taylor $W$, Lye M, et al: Insulin resistance and inflammatory activation in older patients with systolic and diastolic heart failure. Heart 2005, 91:32-37.

13. Bajraktari $G$, Koltai MS, Ademaj $F$, et al: Relationship between insulin resistance and left ventricular diastolic dysfunction in patients with impaired glucose tolerance and type 2 diabetes. Int J Cardiol 2006, 110:206-211.

14. Ingelsson E, Sundstrom J, Arnlov J, et al: Insulin resistance and risk of congestive heart failure. JAMA 2005, 294:334-341.

15. Dinh W, Lankisch $M$, Nickl W, et al: Insulin resistance and glycemic abnormalities are associated with deterioration of left ventricular diastolic function: a cross-sectional study. Cardiovasc Diabetol 2010, 9:63.

16. Boudina S, Abel ED: Diabetic cardiomyopathy revisited. Circulation 2007, 115:3213-3223.

17. Brownlee M: The pathobiology of diabetic complications: a unifying mechanism. Diabetes 2005, 54:1615-1625.

18. Ouwens DM, Diamant M: Myocardial insulin action and the contribution of insulin resistance to the pathogenesis of diabetic cardiomyopathy. Arch Physiol Biochem 2007, 113:76-86.

19. Houstis N, Rosen ED, Lander ES: Reactive oxygen species have a causal role in multiple forms of insulin resistance. Nature 2006, 440:944-948.

20. Palmieri V, Russo C, Bella JN: Treatment of isolated left ventricular diastolic dysfunction in hypertension: reaching blood pressure target matters. Hypertension 2010, 55:224-225.

21. Widlansky ME, Gokce N, Keaney JF Jr, et al: The clinical implications of endothelial dysfunction. J Am Coll Cardiol 2003, 42:1149-1160.

22. Koprowski A, Gruchala M, Rynkiewicz A: Management of left ventricular diastolic heart failure: is it only blood pressure control? Curr Opin Cardiol 2009, 24:161-166.

23. Franklin SS: The importance of diastolic blood pressure in predicting cardiovascular risk. J Am Soc Hypertens 2007, 1:82-93.

24. Bazzano LA, Belame SN, Patel DA, et al: Obesity and left ventricular dilatation in young adulthood: the Bogalusa Heart Study. Clin Cardiol 2011, 34:153-159.

doi:10.1186/1758-5996-5-30

Cite this article as: Tang et al:: The association and predictive value analysis of metabolic syndrome on diastolic heart failure in patients at high risk for coronary artery disease. Diabetology \& Metabolic Syndrome 2013 5:30.

\section{Submit your next manuscript to BioMed Central and take full advantage of:}

- Convenient online submission

- Thorough peer review

- No space constraints or color figure charges

- Immediate publication on acceptance

- Inclusion in PubMed, CAS, Scopus and Google Scholar

- Research which is freely available for redistribution 